JOURNAL OF BUSINESS

and entrepreneurial

studies

\title{
Modelo de Decisión aplicado en la Evaluación y Acreditación de Carreras de Grado
}

\section{Model Decision applied in the Evaluation and Accreditation of Degree Careers}

Ing.Freddy Rolando Villao Santos Ms.C Griffith University

Queensland-Australia fvillaosantos.fvs@gmail.com ORCID https://orcid.org/0000-0003-4282-4924

Ing.Verónica Cecilia Ponce Chalén Ms.C

Universidad de Santa Elena

La libertad-Ecuador veroc_05@hotmail.com ORCID https://orcid.org/0000-0001-5472-4870

Recibido 23 abril 2018 - Aceptado 4 julio - 2018 


\section{Resumen}

La Educación Superior en el Ecuador entró en una etapa de cambios impulsada por las regulaciones que rige el sistema a partir del año 2008, tal situación ha exigido que las Universidades y Escuelas Politécnicas inicien procesos de mejoramiento continuo basados en autoevaluaciones periódicas tomando como referencia diferentes modelos de calidad y en especial el que ha emitido el Consejo de Evaluación, Acreditación y Aseguramiento de la Calidad, CEAACES y que tienen como finalidad acreditarse así como también sus Carreras. El establecimiento de las evidencias que justifican el indicador de calidad, así como el peso que se le da a cada uno es la parte sustancial del proceso de autoevaluación que las instituciones llevan adelante y se hace necesario contar con una herramienta que minimice la subjetividad y generen resultados que en principio pueden ser bueno pero que al final no alcancen para la evaluación externa; por lo que se ha realizado un recorrido por diferentes conceptos, regulaciones y procesos que se aplican en Ecuador para la evaluación de carreras basado en un modelo genérico estableciendo un procedimiento que se ha utilizado para la determinación de los pesos de los indicadores y propone algunas líneas de trabajos futuros.

Palabras clave: Evaluación de carreras, criterios, indicadores de calidad, autoevaluación.

\section{Abstract}

Higher Education in Ecuador entered a stage of changes driven by the regulations that govern the system as of 2008, such situation has required universities and polytechnical schools to start continuous improvement processes based on periodic self-assessments taking as reference different models Of quality and in particular that issued by the Council of Evaluation, Accreditation and Quality Assurance, CEAACES and whose purpose is to accredit as well as their careers. The establishment of the evidence that justifies the quality indicator, as well as the weight given to each is the substantial part of the selfevaluation process that the institutions carry out and it is necessary to have a tool that minimizes subjectivity and generates Results that in principle can be good but that in the end do not reach for the external evaluation; For which it has been made a tour of different concepts, regulations and processes that are applied in Ecuador for the evaluation of careers based on a generic model establishing a procedure that has been used to determine the weights of the indicators and proposes some lines Of future work.

Keywords: Career Assessment, criteria, quality indicators, self appraisal

\section{Introducción}

Tomar una decisión involucra considerar varios criterios y posiblemente estos bajo incertidumbre, la teoría de la decisión nos ayuda con metodologías y herramientas para tratar de dar solución a problemas complejos. 
Estos problemas complejos se presentan en la vida cotidiana prácticamente en todas las actividades del ser humano por lo que las consecuencias de estas decisiones se ven reflejadas a corto, mediano o largo plazo según sea el caso. Se requiere entonces un enfoque objetivo hacia la toma de las decisiones basada en el análisis de datos y la información, esto a su vez constituye uno de los aspectos básicos en un sistema de gestión de la calidad y mejora continua.

Desde este punto de vista y en el ámbito de la educación a partir del año 1990 en Latinoamérica comienzan los primeros esfuerzos entorno a la evaluación de los procesos de enseñanza aprendizaje con miras a asegurar niveles de calidad y excelencia para lo cual los planteamientos en la mejora continua juegan un papel importante.

El Ecuador también entra a formar parte de esta tendencia a partir del año 2007 con procesos de evaluación y acreditación de las universidades que eran parte de su sistema de educación superior.

Para el año 2010 la Asamblea Nacional del Ecuador como órgano legislativo expide la Ley Orgánica de Educación Superior, LOES, que en uno de sus artículos crea los organismos públicos que rigen el Sistema de Educación Superior, entre ellos al CEAACES ${ }^{1}$ organismo que dentro de sus funciones constan la planificación, coordinación y ejecución de los procesos de evaluación,

${ }^{1}$ Consejo de Evaluación, Acreditación y Aseguramiento de la Calidad de la Educación Superior acreditación, clasificación, aseguramiento de la calidad, aprobación de la normativa para estos procesos así como el establecimiento de los criterios e indicadores de calidad como también de los instrumentos a aplicarse.

La Universidad Estatal Península de Santa Elena, UPSE, es una institución de educación superior creada el 22 de julio de 1998 con Registro Oficial No. 366, que ha experimentado tres evaluaciones institucionales y a la fecha se encuentra acreditada en la categoría $\mathrm{C}$ hasta el año 2018 , sin embargo tal como lo establece la $\mathrm{LOES}^{2}$, las instituciones de educación superior deben ser evaluadas $\mathrm{y}$ acreditadas así como sus carreras y programas.

El aseguramiento de la calidad en la educación superior se relaciona con una diversidad de propósitos y marcos metodológicos, que reflejan distintos intereses y necesidades, y, por supuesto, al uso que se dará a los resultados. En general, estos propósitos pueden agruparse en tres grandes categorías, que no son mutuamente excluyentes sino que expresan énfasis dominantes en contextos específicos. Estos son:

El Control de Calidad, expresado en procesos de licenciamiento o autorización de funcionamiento. El control de calidad se identifica con el rol tradicional del Estado de asegurar que la provisión educativa se ajusta a niveles

${ }^{2}$ Ley Orgánica de Educación Superior 
mínimos de calidad. Cuanto los sistemas eran mayoritariamente públicos, esta función se desarrollaba de manera automática, basada en el supuesto (habitualmente correcto) de que la calidad era un rasgo propio de las instituciones públicas.

Cuando los sistemas se diversificaron, y la educación superior quedó en manos de una variedad de proveedores, se hizo indispensable desarrollar mecanismos que permitieran asegurar la operación de instituciones o programas que satisfacían estándares mínimos de calidad.

Así, ante el crecimiento en el número y/o diversidad de las instituciones de educación superior, varios países han desarrollado mecanismos que garantizan que sólo se autorizará la instalación y operación de instituciones o programas que satisfacen ciertos requisitos básicos de calidad. Estos mecanismos llevan distintas denominaciones, pero en la práctica corresponden a lo que puede llamarse licenciamiento o autorización, para indicar que otorgan una licencia básica para la operación como instituciones de educación superior, sobre la base de la verificación de las condiciones en que desarrollan su labor.

La garantía de la calidad, expresado en procesos de acreditación, de carreras o instituciones Aunque cuando se haya logrado asegurar un nivel básico de calidad, subsiste la necesidad de informar a los distintos actores acerca de la calidad de las ofertas educativas. Es necesario, además, dar cuenta pública de los recursos recibidos, ya sea del estado, de los estudiantes o de otras fuentes.

En este caso, el objetivo principal es la provisión de información confiable acerca del grado en que las instituciones o sus programas cumplen con lo que ofrecen y satisfacen las expectativas asociadas a un determinado grupo de referencia, sea éste disciplinario, profesional o laboral. Los sistemas de acreditación evalúan la institución, programa o carrera con relación a sus propios procesos y a un conjunto de estándares, y como consecuencia, dan garantía pública de su calidad, entendida ésta como la capacidad para cumplir tanto con los requerimientos internos (propósitos) como externos (estándares).

E1 Mejoramiento, expresado en procesos de auditoría académica. Este es el objetivo final de todo sistema de aseguramiento de la calidad, aun cuando en general se reconoce que las etapas previas son necesarias. El objetivo de mejoramiento enfatiza el hecho de que la responsabilidad por la calidad corresponde a las instituciones; por tanto, se centra en su capacidad para desarrollar y aplicar políticas y mecanismos eficaces de autorregulación y mejora continua. El procedimiento comúnmente asociado con la mejora es la auditoría académica, en que la evaluación se refiere a los propósitos institucionales y a la forma en que la institución vela por su logro oportuno, y es capaz de hacer los ajustes necesarios cuando detecta debilidades $\mathrm{o}$ áreas deficitarias. 
América Latina está entre las regiones pioneras en el campo del aseguramiento de la calidad, considerando que con excepción de Estados Unidos, se trata de procesos relativamente jóvenes. En efecto, ya durante la década de los noventa, al menos Chile, Colombia y Argentina contaban con procesos en diversas etapas de consolidación. En Europa, en cambio, si bien en esos años existían procesos de evaluación, la acreditación comenzó a desarrollarse después del 2000, el mismo período en que comenzaron a desarrollarse procesos en Australia y varios países del Asia.

\section{Materiales y métodos}

El modelo busca tomar en cuenta de forma integral todos los aspectos involucrados en la actividad universitaria por esta razón se basa en la metodología multicriterio.

El CEAACES justificó la utilización de esta metodología basada en:

$\checkmark$ Investigación e integración de intereses y objetivos de múltiples actores.

$\checkmark$ Complejidad de la configuración de los múltiples actores.

$\checkmark$ Método de alternativas de valoración.

$\checkmark$ Objetividad e inclusión de diferentes intereses de los actores.

Para el desarrollo del modelo se siguió la siguiente metodología:
Identificación de los criterios principales e indicadores a ser medidos.

$\checkmark$ Diseño de guías de verificación in situ $\checkmark$ Aplicación de plataforma informática para el ingreso de información

$\checkmark$ Diseño de las funciones de utilidad

$\checkmark$ Definición de indicadores con mayor relevancia

$\checkmark$ Asignación de pesos relativos por criterios, subcriterios e indicadores

$\checkmark$ Análisis de funciones de utilidad

$\checkmark$ Suma ponderada entre las utilidades de cada indicador para la valoración de los subcriterios.

Esta metodología sirve para tratar problemas de toma de decisiones en los que se presentan "diversos objetivos o criterios que simultáneamente deben incorporarse". (Ávila, 2000)

A partir de lo mencionado y para la aplicación de la metodología multicriterio se ha establecido los siguientes criterios: Pertinencia, Plan Curricular, Academia, Ambiente Institucional y Estudiantes.

Criterio Pertinencia: evalúa que la carrera o programa académico, responda a las expectativas y necesidades de la sociedad, a la planificación nacional, a la prospectiva de desarrollo científico, humanístico y tecnológico mundial y a la diversidad cultural.

Criterio Plan Curricular: evalúa los distintos niveles de concreción del currículo asegurando coherencia e 
integración entre el macro, meso y micro-currículo.

Criterio Academia: evalúa a los profesores de la carrera. Los profesores son el personal encargado directamente del proceso de enseñanza aprendizaje, de la investigación y la vinculación, quienes deben tener las competencias necesarias para cumplir todas las áreas curriculares de la carrera.

Criterio Ambiente Institucional: evalúa el sistema de gestión académica de la carrera ejecutado por una coordinación / dirección responsable de los procesos de seguimiento académico curricular y de recursos de apoyo.

Criterio Estudiantes: evalúa la participación estudiantil en actividades complementarias, tutorías, políticas de bienestar estudiantil y el proceso de acreditación de las carreras.

La metodología utilizada para la evaluación de carreras con la aplicación del modelo propuesto por el ente evaluador, propone una asignación de valoraciones de desempeño (utilidades) a cada uno de los indicadores del modelo; esta asignación se efectúa de acuerdo con ciertas funciones de utilidad. Para la aplicación de lo mencionado en el caso de los indicadores cualitativos, se tiene:

1 para el nivel satisfactorio, $\checkmark 0,5$ para medianamente satisfactorio y $\checkmark 0$ para deficiente.
En el caso de los indicadores cuantitativos y mixtos, se tiene una función matemática que adjudica un valor de desempeño entre 0 y 1 .

Para obtener un resultado final de la carrera en el entorno de aprendizaje se utiliza una suma ponderada de los desempeños de todos los indicadores.

La determinación de las variables es la primera acción que se toma puesto que en base a estas variables se identifican los indicadores que son parte del modelo. Estas variables son calculadas a partir de los datos que se obtienen directamente de las carreras.

Para el caso de los indicadores cualitativos la variable y el indicador coinciden.

El modelo de evaluación del entorno de aprendizaje de la carrera utilizado para nuestra aplicación tiene un total de 37 indicadores de los cuales 17 son cuantitativos y 20 cualitativos.

\section{Resultados}

El modelo genérico que propone el Consejo de Evaluación, Acreditación y Aseguramiento de la calidad para la evaluación de carreras, contiene una serie de variables de carácter discrecional, que combinada a las variables cuantitativas generan una utilidad que al final del ejercicio genera un resultado cuyo peso está más ligado al criterio personal que al dato cuantificable. Se requiere demostrar cómo se puede minimizar el impacto de 
una evaluación basada en criterio del evaluador y utilizar otro mecanismo para dar valor a la variable.

El modelo en estudio contempla una serie de evidencias aceptables como cumplimiento del indicador en su respectiva variable, la discrecionalidad con que se juzga los indicadores cualitativos en comparación con lo exacto de los cuantitativos merece una reflexión de que es mejor sin que eso afecte la calidad.

\section{Modelo de evaluación para Carreras.}

La legislación del país determina que la acreditadora del Estado es el ente que certifica la calidad de los programas, carreras y la institución en su conjunto, sería muy importante desde el Sistema de Educación Superior, poder generar un modelo que permita valorar la calidad y aplicarlo como estrategia de mejora continua y que sus resultados permitan cumplir estándares no solo nacionales sino internacionales.

\section{Algoritmos de medición de calidad}

Indudablemente que cada una de las acciones que se realicen deben tener un mayor o menor grado de aproximación al valor exacto, sin embargo, queda en el debate si el instrumento con el que se mide aporta, la forma como se asigna la utilidad es pertinente, los niveles de agrupamiento son consistentes. En el año 2010, luego de la evaluación que realizará el CONEA a las universidades hubo muchos cuestionamientos al modelo aplicado y de a poco se ha ido mejorando, sería importante valorar la eficacia del modelo y compararlo con otros similares en sus fines.

\section{Discusión}

Comportamiento de variables cuantitativas y cualitativas que propone el Consejo de Evaluación, Acreditación y Aseguramiento de la Calidad para la evaluación de carreras, contiene una serie de variables de carácter discrecional, que combinada a las variables cuantitativas generan una utilidad que al final del ejercicio genera un resultado cuyo peso está más ligado al criterio personal que al dato cuantificable. Se requiere demostrar cómo se puede minimizar el impacto de una evaluación basada en criterio del evaluador y utilizar otro mecanismo para dar valor a la variable.

El modelo en estudio contempla una serie de evidencias aceptables como cumplimiento del indicador en su respectiva variable, la discrecionalidad con que se juzga los indicadores cualitativos en comparación con lo exacto de los cuantitativos merece una reflexión de que es mejor sin que eso afecte la calidad.

\section{Conclusión}

Modelo de evaluación para carreras en la legislación del país determina que la acreditadora del Estado es el ente que certifica la calidad de los programas, carreras y la institución en su conjunto, 
sería muy importante desde el Sistema de Educación Superior, poder generar un modelo que permita valorar la calidad y aplicarlo como estrategia de mejora continua y que sus resultados permitan cumplir estándares no solo nacionales sino internacionales.

Los algoritmos de medición de calidad indudablemente que cada una de las acciones que se realicen deben tener un mayor o menor grado de aproximación al valor exacto, sin embargo, queda en el debate si el instrumento con el que se mide aporta, la forma como se asigna la utilidad es pertinente, los niveles de agrupamiento son consistentes. En el año 2010, luego de la evaluación que realizará el CONEA a las universidades hubo muchos cuestionamientos al modelo aplicado y de a poco se ha ido mejorando, sería importante valorar la eficacia del modelo y compararlo con otros similares en sus fines.

\section{Referencias}

[1] Ávila, R. (2000). El AHP (Proceso Analítico Jerárquico) y su aplicación para determinar los usos de las tierras. Santiago de Chile.

[2] Aznar Bellver, J. (2012). Nuevos Métodos de Valoración Modelos Multicriterio. Valencia: Editorial Universitat Politécnica de Valencia.

[3] Bárcena, A. (2013). Estudio Económico de América Latina y el
Caribe. Santiago de Chile: Naciones Unidas.

[4] Bernal, S., Martínez, M., Parra, A., \& Jiménez, J. (2015). Investigación Documental Sobre Calidad De La Educación En Instituciones Educativas del Contexto Iberoamericano. Entramados - Ecuación y Sociedad.

[5] Botero, C. (2009). Cinco Tendencias de la Gestión Educativa. Revista Iberoamericana de Educación.

[6] CEAACES. (2012). Modelo Genérico para la Evaluación de Universidades.

[7] CEAACES. (2013). Informe General sobre la Evaluación, Acreditación y Categorización de las Universidades y Escuelas Politécnicas.

[8] CEAACES. (2015). Modelo genérico de evaluación del entorno del aprendizaje de carreras presenciales y semipresenciales de las Universidades y Escuelas Politécnicas del Ecuador. Ecuador.

[9] CES. (Noviembre de 2013). Reglamento Régimen Académico del Sistema Nacional de Educación Superior. San Francisco de Quito, Ecuador.

[10] CINDA. (2007). Eduación Superior en Iberoamérica, Informe 2007. Santiago de Chile: RIL*Editores. 
[11] Ecuador. (2008). Constitución de la República del Ecuador. Registro Oficial No. 449.

[12] Ecuador. (2010). Ley Orgánica de Eduación Superior. Registro Oficial No. 298.

[13] García, E. G. (1999). Evaluación y Calidad de la Universidad: El proyecto de la Universidad Complutense de Madrid. Revista Electrónica Interuniversitaria de Formación del Profesorado. 